\title{
Subtle errors of bladder wall thickness measurement have a significant impact on the calculation of ultrasound-estimated bladder weight. A pilot study
}

\author{
Vasileios I. Sakalis' ${ }^{1}$, Vasileios Sfiggas ${ }^{2}$, Ioannis Vouros ${ }^{2}$, Georgios Salpiggidis ${ }^{2}$, \\ Athanasios Papathanasiou², Apostolos Apostolidis ${ }^{3}$
}

${ }^{1}$ Department of Urology, General Hospital of Thessaloniki, Agios Pavlos, ${ }^{2}$ Department of Urology, General Hospital of Thessaloniki, Hippokrateion, ${ }^{3}$ Department of Urology, $2^{\text {nd }}$ University Hospital of Thessaloniki, Papageorgiou, Thessaloniki, Greece.

\begin{abstract}
Aims: Ultrasound-estimated bladder weight (UEBW), is an emerging diagnostic tool, which has been used in both males and females with lower urinary tract dysfunction. The currently acknowledged UEBW calculation methods rely on the accurate measurement of bladder wall thickness (BWT). We aim to identify if subtle errors in BWT measurement have a significant impact on UEBW calculations. Materials and methods: Twenty patients were randomly selected from an overactive bladder patient cohort. The primary endpoint was to identify the range of false BWT measurements outside which significant changes in UEBW calculation occur. We used the Kojima method and a semi-automatic 3-D model that is based on Chalana's principle. Measurements were performed using the correct BWT and a series of faulty calculations from $+0.5 \mathrm{~mm}$ to $-0.5 \mathrm{~mm}$ using steps of $0.05 \mathrm{~mm}$ from true BWT. The effect of a fixed $0.5 \mathrm{~mm}$ BWT error was checked in bladder volumes above and below $250 \mathrm{ml}$ and in three UEBW groups ( $<35$ gr; $36-50$ gr; $>51$ gr). Results: BWT measurement errors above 0.25 mm cause statistically significant changes in UEWB calculation when a 3-D model is used and errors above $0.15 \mathrm{~mm}$ when Kojima's method is used. At a fixed BWT error of $0.5 \mathrm{~mm}$ and bladder volume $<250 \mathrm{ml}$, there is a $23.76 \%$ deviation from true UEBW, while at volumes $>250 \mathrm{ml}$ the deviation is $32.72 \%$. The deviation is inversely proportional to the UEBW result, and heavier bladders deviate less. Conclusions: UEBW is a promising diagnostic tool, but small errors in BWT measurement might cause significant deviation from the true values. A 3-D calculation model appears to minimize such risks.
\end{abstract}

Keywords: ultrasound; bladder weight; bladder wall thickness; error

\section{Introduction}

Over the past decade, the interest for bladder wall thickness (BWT) and detrusor wall thickness (DWT) measurements has grown rapidly. Their potential use as add-ons to uroflowmetry and conventional ultrasonography, has initiated a quest for their diagnostic validity in

Received 10.12.2017 Accepted 10.04.2018

Med Ultrason

2018, Vol. 20, No 3, 292-297

Corresponding author: Vasileios I. Sakalis MSc, FEBU, FRCS

Department of Urology, General Hospital of

Thessaloniki, Agios Pavlos,

161 Ethnikis Antistasis, Thessaloniki,

Greece, POB 55134

Phone: +30 2313 304676, Mob: +30 6987402020

E-mail: vsakkalis@hotmail.com different types of lower urinary tract problems in both genders $[1,2]$. Even if formal standardization is still lacking, numerous studies have been published so far, using these modalities for diagnostic purposes and for the assessment of treatment efficacy. It has been proved that a urodynamic diagnosis of detrusor overactivity (DO) correlates well with BWT and DWT measurements [36]. A small study could not distinguish between DO and bladder outlet obstruction (BOO) in males [1]. A recent meta-analysis on non invasive diagnostic tests in males with lower urinary tract symptoms (LUTS), did not find any threshold or cut-off value that can be safely used [7].

The principle of BWT and DWT measurement is that muscle thickening should compensate the increased peripheral resistances, in a similar manner to the cardiac muscle, as a consequence of an abnormally high after- 
load $[8,9]$. Morphological studies have shown that the thickening is a dual effect of smooth muscle hypertrophy and collagen deposition [8]. Animal studies have demonstrated bladder wall hypertrophy and hence increased bladder weight following partially induced $\mathrm{BOO}$, within as little as 2 weeks [10].

The bladder wall appears on ultrasound (US) as a three-layered structure with the hypoechoic detrusor between the two hyperechoic layers that represent the mucosa and the serosa [1]. Various techniques have been proposed to measure BWT and DWT, using different approaches (transvaginal, transperineal or suprapubic), with different probes $(3.5 \mathrm{MHz}, 5 \mathrm{MHz}$, or $7.5 \mathrm{MHz})$ in either an empty bladder or in comfortable filling volumes [11]. The original approach in females measured BWT transvaginally at three areas with empty bladder, and the BWT was the average of these measurements [12]. In men, Oelke et al proposed a method using a $7.5 \mathrm{MHz}$ probe suprapubically which measures anterior bladder wall thickness at $250 \mathrm{ml}$ filling volume [13].

Ultrasound-estimated bladder weight (UEBW) concept has been advocated as a more reliable measure of bladder wall hypertrophy, because it can be calculated regardless of the degree of bladder filling. UEBW has been used as a diagnostic tool in males or females with LUTS, as a predictor of the outcome or as a monitoring tool [1].

Up to date, there are two methods for UEBW calculation. The Kojima method considers the bladder as a perfect sphere and calculates UEBW when bladder volume and BWT are known [14]. The Chalana method, which is an automatic measurement from 3-Dimensional (3-D) US, based on the calculation of bladder surface [3]. Although their principle of measurement differs, they both rely on an accurate BWT measurement. The majority of the relevant publications are based on Chalana's principle, which lacks formal standardization.

Cut-off values for BWT, DWT, and UEBW are not strictly set. None of the studies published so far have quantified the measurement error and how it influences the final values of the analysed parameters. In this pilot study, we aim to investigate if subtle errors of BWT measurement affect the UEBW calculation and to assess the level of the BWT error which does not produce statistically significant changes in UEBW.

\section{Materials and methods}

\section{Patient sample}

Twenty male patients were randomly selected from an overactive bladder patient cohort of more than onehundred fully investigated patients with cysto-manometry and US studies, which were performed before and after combined pharmacologic intervention with an alpha blocker (Tamsulosin) and an anticholinergic (Solifenacin). It is a homogenous group of patients, with marked storage-type LUTS (mainly urgency, possibly accompanied by micturition frequency, nocturia and/or urgency-associated incontinence), prostate volume $>30$ $\mathrm{ml}$, postvoid residual $<100 \mathrm{ml}$, prostate specific antigen $<4 \mathrm{ng} / \mathrm{ml}$, maximum flow rate $>10 \mathrm{ml} / \mathrm{sec}$, without neurological problems and free of urinary tract infections or urinary tract malignancies. We used the General Electronic Logic E9 ultrasound device, equipped with a 3.5 $\mathrm{MHz}$ and an $8.0 \mathrm{MHz}$ transabdominal and a $7.5 \mathrm{MHz}$ transrectal probes. All measurements were performed by two physicians and the results were the means of the two measurements. All patients gave their informed consent, according to the World Medical Association Declaration on Helsinki, revised in 2000, Edinburgh. The study was approved by the local Ethics Committee.

The primary endpoint of this study was to identify the arithmetic range of BWT measurements above which statistically significant changes in UEBW calculation occur. Secondary end-points were the comparison of subtle errors effect in different UEBW groups, in different volumes of the bladder filling and between the two available techniques. For this reason, we evaluated sequential calculations of UEBW, using false BWT values with an increment $0.05 \mathrm{~mm}$ above and below the true BWT value. We used the Kojima method or spheroidal model and a 3-D method which is based on Chalana's principle [3]. This 3-D method is a semi-automatic calculator of UEBW that allows the user to interfere with multiple parameters such as BWT, bladder volume and bladder surface.

\section{Measurement of bladder wall thickness}

BWT was measured suprapubicaly using the 8.0 $\mathrm{MHz}$ probe. This frequency can image pelvic organs with satisfactory resolution $(<0.13 \mathrm{~mm})$, including the anterior bladder wall even in obese individuals $[13,15]$. A single perpendicular focused image showing 2 hyperechoic lines, representing the mucosa and the adventitia, and a middle hypoechoic area representing the detrusor muscle were considered adequate (fig 1) [13]. According to the standardization of International Continence Society (ICS) on imaging, BWT represents the average of three measurements on the anterior bladder wall with the bladder filled at more than $50 \%$ of normal capacity or more than $250 \mathrm{ml}[13,16]$. False measurements of transversalis fascia echo or median umbilical ligament echo were avoided [13]. Since many of our subjects presented with small bladder volumes, the BWT was measured at the level of normal desire of bladder fullness. 


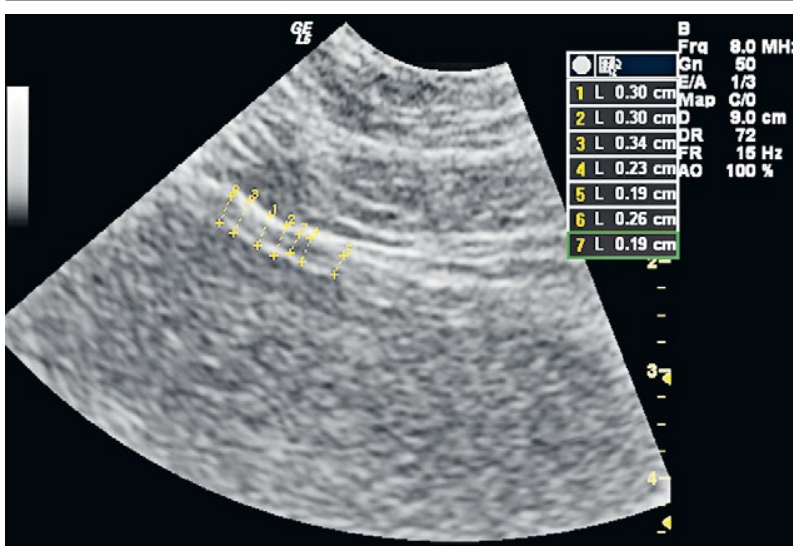

Fig 1. Ultrasonographic image of bladder wall using an 8.0 $\mathrm{MHz}$ probe and measurement of bladder wall thickness (measurements 1-3) and detrusor wall thickness (measurements 4-7).

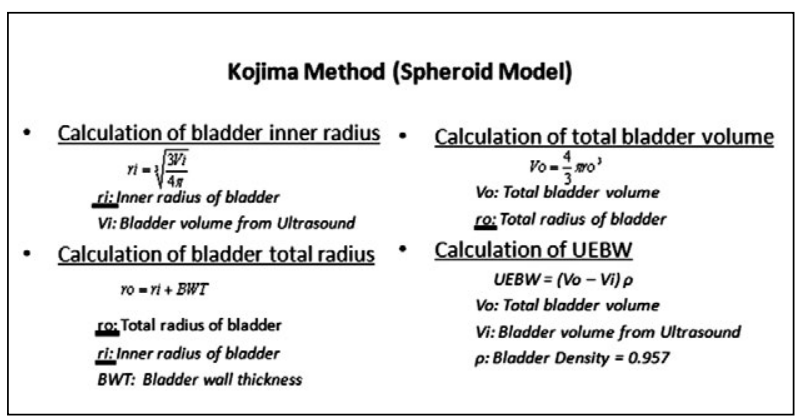

Fig 2. The mathematical calculations of spheroidal model of Kojima.

\section{Measurement of ultrasound estimated bladder weight by the Kojima method}

On the assumption that the bladder is a perfect sphere, Kojima et al estimated bladder weight when volume and BWT are known [14]. The respective calculations in our patient sample are shown in figure 2.

\section{Measurement of ultrasound estimated bladder weight using the 3-D bladder Model based on Chalana's principle}

According to the ICS subcommittee on imaging standardisation, "the most accurate technique for measuring the volume of an irregular object is the step sectioning and integration of the area on each side' [1]. This step-sectioning or planimetric volumetric approach was first described by Watanabe et al in 1982 [17]. It involves the volume calculation of each separate slice and the total volume of the organ under evaluation, results from the sum of each separate volumetry. The technique is implemented in various measurements, such as volume measurements of the urinary bladder, prostate gland, renal parenchyma, and the cardiac left ventricle from ultrasound images.
A 3.5 MHz probe was used to acquire images for further analysis. The probe was placed $2 \mathrm{~cm}$ above pubis symphysis and the bladder was scanned from the bladder neck to the dome to obtain parallel images by a step of $8 \mathrm{~mm}$, using a stabilization stepper (Accucare by Multipurpose Workstation) designed for transperineal template biopsies, which has been modified to hold the convex probe as well. This distance was selected to reduce the investigation time and patient's discomfort. A smaller step size would lead to a larger amount of images, increase the investigation time, and potentially be negatively influenced by the subject's movements, while a larger step size would reduce the available information. The series of planimetric images were saved in DICOM format for further analysis.

For each bladder section image, the inner bladder contour was manually delineated and segmented. Considering the step length $(8 \mathrm{~mm})$, a small cylinder was triangulated for every section/step using MATLAB R2012a (Matrix Laboratory, Mathworks) and using $\mathrm{C}++$ computing language a 3D model was developed [18]. The bladder surface area (BSA) was calculated by adding the triangulated surface area of each cylinder. The UEBW was calculated from the following formula UEBW=BSA x BWT x $\rho$, where $\rho$ is the density of the bladder [14].

\section{Details of the comparison processing}

For each patient, we calculated the true UEBW, based on the true BWT measurement, using both techniques Kojima's and 3D model. Then, 20 additional false UEBW measurements were performed using incorrect BWT measurements at $0.05 \mathrm{~mm}$ step apart from the true BWT, 10 of them with a positive increment up to +0.5 $\mathrm{mm}$ and another 10 with a negative step down to -0.5 $\mathrm{mm}$. At the end, each patient had 21 UEBW calculations using the Kojima method and 21 UEBW calculations using the 3-D model. The Kojima and 3-D simulations were automated on MATLAB (Matrix Laboratory, Mathworks) [18]. Mean percentage deviation from true UEBW calculation was assessed.

Each patient was then allocated to one of three groups based on UEBW: Group I (UEBW up to $35 \mathrm{gr}$ ), Group II (UEBW 36 - 50 gr), and Group III (UEBW more than 51 gr). The mean percentage deviation was calculated for each group. In addition, the patients were allocated to two groups, based on the initial bladder volume (Group 1: Bladder Volume $<250 \mathrm{ml}$, Group 2: Bladder Volume $>250 \mathrm{ml}$ ).

\section{Statistical analysis}

The statistical analysis was performed using SPPS 20.0 (IBM Corporation). Intragroup variability was assessed by t-test and intergroup variability by MannWhitney test. A baseline value was considered as the 
true UEBW calculation, when the true BWT was used. Statistical significance was set at $\mathrm{p}<0.05$ values.

\section{Results}

The baseline characteristics of the study group are shown in table I. Mean bladder volume at examination was $242.17 \mathrm{ml}$ (range 100-528 ml). Using sequential calculations of UEBW with incorrect BWT measurements with a $0.05 \mathrm{~mm}$ increment step from true BWT, we found that errors up to $0.2 \mathrm{~mm}$ in BWT measurement do not cause significant changes in the 3-D calculation of UEBW. An error of $0.25 \mathrm{~mm}$ or more, leads to a statistically significant difference $(p<0.001)$ from the true value of UEBW, with a mean difference of $4.08 \%$. It seems that the range of error in BWT measurement to avoid incorrect UEBW measurements is from -0.2 $\mathrm{mm}$ to $+0.2 \mathrm{~mm}$. Using the Kojima method, incorrect BWT measurements from $0.15 \mathrm{~mm}$ above or below the true BWT produce significantly different UEBW results (table II).

Table III, compares the mean percentage deviations from true UEBW measurement (baseline) in different UEBW groups, when there is error in the BWT measurement at $0.5 \mathrm{~mm}$. Both groups show that deviation is inversely proportional to the UEBW value and heavier bladders tend to deviate less. 3-D UEBW calculation deviates less compared to the Kojima method but both are statistically significantly different from baseline $(p<0.05)$. At the extreme values, the heaviest bladder weighed $115.73 \mathrm{gr}$, with the 3-D model showing a mean deviation of $17.54 \%$, an increment deviation of $7.89 \%$ and a decrement deviation of $8.74 \%$. On the other extreme, the lightest bladder weighed $22.42 \mathrm{gr}$, with the 3 -D model showing a mean deviation of $32.25 \%$, an increment deviation of $14.52 \%$ and a decrement deviation of $17.65 \%$.

Table I. Baseline characteristics of the study group

\begin{tabular}{ll}
\hline & Mean (range) \\
\hline Age (years) & $70.25(49-85)$ \\
Bladder volume (ml) & $242.17(100-528)$ \\
BWT (mm) & $3.065(1.4-5.2)$ \\
DWT (mm) & $1.735(0.7-3.825)$ \\
UEBW Kojima (gr) & $53.32(29.3-125.70)$ \\
UEBW 3-D (gr) & $47.74(22.4-115.7)$ \\
Volume prostate (ml) & $38.34(30-83.9)$ \\
PSA (ng/ml) & $2.19(0.2-4.0)$ \\
Uroflow (ml/sec) & $13.26(10-24)$ \\
BOOI & $63.88(12-164.8)$ \\
BCI & $134.97(63-218)$ \\
IPSS & $21.16(9-28)$ \\
\hline
\end{tabular}

BWT: Bladder wall Thickness; DWT: Detrusor wall thickness; UEBW: Ultrasound-estimated bladder weight; PSA: Prostate Specific Antigen; BOOI: Bladder outlet obstruction index; BCI: Bladder contractility Index; IPSS: International prostate symptoms score

Table II. Comparison of the mean percentage deviation based on the arithmetic deviation from the true BWT.

\begin{tabular}{|c|c|c|c|c|}
\hline & Mean (SD) Kojima & P value & Mean (SD) Planimetry & P value \\
\hline BWT -0.5 & $43.42(19.87)$ & 0.001 & $39.58(18.57)$ & 0.001 \\
\hline BWT -0.45 & $44.40(20.01)$ & 0.001 & $40.23(18.74)$ & 0.001 \\
\hline BWT -0.4 & $44.54(20.69)$ & 0.001 & $41.21(18.86)$ & 0.001 \\
\hline $\mathrm{BWT}-0.35$ & $45.88(20.25)$ & 0.001 & $41.56(18.74)$ & 0.001 \\
\hline $\mathrm{BWT}-0.3$ & $47.37(20.45)$ & 0.001 & $42.84(19.17)$ & 0.001 \\
\hline $\mathrm{BWT}-0.25$ & $48.36(20.61)$ & 0.001 & $43.66(19.33)$ & 0.001 \\
\hline $\mathrm{BWT}-0.2$ & $49.37(20.77)$ & 0.001 & $44.48(19.49)$ & 0.059 \\
\hline $\mathrm{BWT}-0.15$ & $50.35(20.94)$ & 0.001 & $45.29(19.66)$ & 0.092 \\
\hline $\mathrm{BWT}-0.1$ & $51.35(21.10)$ & 0.291 & $46.11(19.84)$ & 0.388 \\
\hline BWT -0.05 & $52.79(21.25)$ & 0.678 & $46.93(20.02)$ & 0.548 \\
\hline True BWT & $53.36(21.46)$ & - & $47.74(20.20)$ & - \\
\hline $\mathrm{BWT}+0.05$ & $52.81(22.38)$ & 0.724 & $48.56(20.39)$ & 0.467 \\
\hline $\mathrm{BWT}+0.1$ & $55.37(21.82)$ & 0.342 & $49.37(20.58)$ & 0.201 \\
\hline $\mathrm{BWT}+0.15$ & $55.24(21.15)$ & 0.001 & $49.69(21.16)$ & 0.112 \\
\hline $\mathrm{BWT}+0.2$ & $57.39(22.21)$ & 0.001 & $51.00(20.98)$ & 0.081 \\
\hline $\mathrm{BWT}+0.25$ & $58.41(22.41)$ & 0.001 & $51.83(21.18)$ & 0.001 \\
\hline $\mathrm{BWT}+0.3$ & $59.43(22.61)$ & 0.001 & $52.64(21.39)$ & 0.001 \\
\hline $\mathrm{BWT}+0.35$ & $60.45(22.81)$ & 0.001 & $53.46(21.60)$ & 0.001 \\
\hline $\mathrm{BWT}+0.4$ & $61.48(23.02)$ & 0.001 & $54.67(21.82)$ & 0.001 \\
\hline $\mathrm{BWT}+0.45$ & $63.00(23.15)$ & 0.001 & $55.09(22.04)$ & 0.001 \\
\hline $\mathrm{BWT}+0.5$ & $63.48(23.44)$ & 0.001 & $55.31(22.48)$ & 0.001 \\
\hline
\end{tabular}

BWT: Bladder wall thickness (mm), SD: Standard Deviation 
There is a statistically significant difference in the mean deviation when bladder volume is considered. Table IV, shows the comparison for volumes up to $250 \mathrm{ml}$ and $250 \mathrm{ml}$ or more when there is an error in BWT at $0.5 \mathrm{~mm}$. It appears that deviation is proportional to filling volumes. Mean percentage deviation from true UEBW measurement at $0.5 \mathrm{~mm}$ BWT error was $23.76 \%$ in bladder volumes less than $250 \mathrm{ml}$ and $32.72 \%$ in bladder volumes higher than $250 \mathrm{ml}$. Both changes were significantly different from baseline $(\mathrm{p}<0.01)$.

\section{Discussions}

This study has shown that subtle changes of BWT measurement may significantly affect UEBW calculation. Both techniques used showed a significant $(\mathrm{p}<0.001)$ deviation from baseline in UEBW calculation, when a BWT error from the true value is $0.25 \mathrm{~mm}$ or more for the 3-D calculation and $0.15 \mathrm{~mm}$ or more for the Kojima method. We thus assume that subtle errors up to $0.15 \mathrm{~mm}$ are 'safe' and do not cause statistical significant changes in UEBW calculation with either method. As expected, Kojima's spheroidal method is more sensitive to small BWT changes when compared to the 3-D method due to the volume formula effect that raises BWT measurement to the third power [6].

We have also demonstrated that thicker and heavier bladders tend to deviate less from true values. This is due to the percentage changes, since an error of $0.5 \mathrm{~mm}$ in BWT measurement of a $5 \mathrm{~mm}$-thick wall represents a $10 \%$ deviation while the same error in a $2 \mathrm{~mm}$ thick wall represents a $25 \%$ deviation. Table III shows that a 0.5 $\mathrm{mm}$ error in BWT causes $27.89 \%$ deviation in a heavy bladder with UEBW $>51 \mathrm{gr}$, while the same error causes $33.6 \%$ deviation in a light bladder weighing $<35$ gr. These deviations are proportional with smaller errors. At $0.5 \mathrm{~mm}$ BWT error there is $12.55 \%$ deviation in UEBW $>51 \mathrm{gr}$ and $15.11 \%$ deviation in UEBW $<35$ gr.

Bladder filling volumes affect also the true UEBW. Oelke et al found that BWT is stable at filling volumes $250 \mathrm{ml}$ to $400 \mathrm{ml}$ [11]. Therefore, any deviation from baseline measurements could be attributed to volume changes. The Kojima method is affected by volume changes as one gathers by its calculation formula. The 3-D method is affected less, since it uses the triangulated bladder surface area. Yet, subtle changes $(0.5 \mathrm{~mm})$ in BWT measurement cause a $32.72 \%$ deviation from the true UEBW value when the 3-D method is applied in volumes higher than $250 \mathrm{mls}$ and $23.76 \%$ deviation at volumes less than $250 \mathrm{ml}$. These changes are significant from baseline ( $\mathrm{p}=0.017$ and $\mathrm{p}=0.024$ respectively).

Automatic measurement systems have several advantages [19]. They are user-friendly, hand-held devices, typically used by non-specialized individuals, and reduce the need for sophisticated calculations. It should be noted that the vast majority of studies including UEBW are on

Table III. Comparison of the mean percentage deviation in different groups based on bladder weight (Group I UEBW up to 35gr; Group II UEBW 36-50 gr; Group III UEBW more than 51 gr), with an error of BWT measurement at $0.5 \mathrm{~mm}$.

\begin{tabular}{lllll}
\hline & & Group I $(\mathbf{n}=\mathbf{6})$ & Group II $(\mathbf{n}=\mathbf{8})$ & Group III (n=6) \\
\hline Kojima & MPD (range) & $39.1^{*}(30.71-53.65)$ & $33.46^{*}(27.85-38.47)$ & $28.71^{*}(21.56-46.52)$ \\
& SD & 8.87 & 4.43 & 10.64 \\
& MPD increment & $17.86^{*}$ & $15.27^{*}$ & $13.1^{*}$ \\
& MPD decrement & $22.49^{*}$ & $18.32^{*}$ & $15.57^{*}$ \\
& MP model & $33.59^{*}(26.32-52.63)$ & $31.64^{*}(25.64-37.03)$ & $25.22^{*}(15.6-45.45)$ \\
& SD & 10.86 & 4.78 & 12.53 \\
& MPD increment & $15.11^{*}$ & $14.24^{*}$ & $10.81 \$$ \\
& MPD decrement & $19.01^{*}$ & $17.32^{*}$ & $14.12 \$$ \\
\hline
\end{tabular}

* $\mathrm{p}<0.01$ from True UEBW calculation; $\$ \mathrm{p}=0.035$ from True UEBW calculation; $\mathrm{n}$ - number of patients; BWT: Bladder Wall thickness; UEBW: Ultrasound Estimated Bladder weight; SD: standard deviation; MPD: mean percentage deviation (\%)

Table IV. Comparison of the mean percentage deviation in UEBW calculation based on bladder volume with an error in BWT measurement at $0.5 \mathrm{~mm}$

\begin{tabular}{llll}
\hline & & \multicolumn{2}{l}{ Bladder Volume } \\
\cline { 3 - 4 } & & $<\mathbf{2 5 0} \mathbf{~ m l} \mathbf{~ ( n = 1 2 )}$ & $>\mathbf{2 5 0} \mathbf{~ m l ~ ( n = 8 )}$ \\
\hline Range $(\min -\max )$ & & $80-240 \mathrm{ml}$ & $260-528 \mathrm{ml}$ \\
Kojima & MPD (range) & $27.01^{*}(21.56-33.47)$ & $35.76^{*}(19.40-53.64)$ \\
3D model & MPD (range) & $23.76^{*}(15.61-32.26)$ & $32.72 *(17.54-52.63)$ \\
\hline
\end{tabular}

$* \mathrm{p}<0.01$ from True UEBW calculation; MPD: mean percentage deviation (\%); BWT: Bladder Wall thickness; UEBW: Ultrasound Estimated Bladder weight, $\mathrm{n}$ - number of patients 
Bladder Scan Verathon system. According to the official manual, Bladder scan can measure bladders filled from 100 to $300 \mathrm{ml}$ and BWT from 1 to $4 \mathrm{~mm}$, thus excluding patients with a thicker bladder wall as well as those with very small or very large bladder capacity as for example in detrusor overactivity patients. A disadvantage is the use of a 3.5 $\mathrm{MHz}$ probe which does not have adequate discriminative penetration. These issues were raised by Oelke et al, in their comparison of a hand measurement to the automatic measurements of BWT [19]. We can add to this knowledge that BWT needs to be carefully assessed to have a true UEBW measurement that can be safely reproduced.

The strengths of this study include the homogeneous sample of subjects and the simulation on these measurements with two different techniques that are widely used in the literature. A limitation, is that we did not use the prototype BladderScan machine, since it was impossible to run multiple faulty values for a single patient. Instead, we developed a semi-automatic model for this purpose that is based on the same principle, serving the purpose of this study.

\section{Conclusion}

Results of this pilot study suggest that UEBW could become a useful tool for diagnostic and monitoring purposes in patients with LUTS, especially in patients with overactive bladder syndrome, since their thicker bladder walls and their small bladder capacity minimize the risk for inaccurate measurements. The impact of subtle changes in BWT measurement at the level of $0.2 \mathrm{~mm}$ for 3 -D calculation and at level $0.1 \mathrm{~mm}$ for Kojima calculation of UEBW should be considered.

Acknowledgements: The authors would like to thank Dr Anastasia Gkotsi, for her contribution to the statistical analysis of this project.

\section{Conflict of interest: none}

\section{References}

1. Housami F, Drake F, Abrams P. The use of ultrasound-estimated bladder weight in diagnosing bladder outlet obstruction and detrusor overactivity in men with lower urinary tract symptoms. Indian J Urol 2009;25:105-109.

2. Oelke M, Hofner K, Jonas U, de la Rosette JJ, Ubbink DT, Wijkstra H. Diagnostic accuracy of noninvasive tests to evaluate bladder outlet obstruction in men: detrusor wall thickness, uroflowmetry, postvoid residual urine, and prostate volume. Eur Urol 2007;52:827-834.

3. Chalana V, Dudycha S, Yuk JT, McMorrow G. Automatic measurement of ultrasound estimated bladder weight
(UEBW) from Three-Dimensional ultrasound. Rev Urol 2005; 7:S22-S28.

4. Khullar V, Cardozo LD, Salvatore S, Hill S. Ultrasound: a non-invasive screening test for detrusor instability. Br J Obstet Gynaecol 1996;103:904-908

5. Robinson D, Anders K, Cardozo L, Bidmead J, Toozs-Hobson P, Khullar V. Can ultrasound replace ambulatory urodynamics when investigating women with irritative urinary symptoms? BJOG 2002;109:145-148.

6. Serati M, Salvatore S, Cattoni E, Soligo M, Cromi A, Ghezzi F. Ultrasound measurement of bladder wall thickness in different forms of detrusor overactivity. Int Urogynecol J 2010;21:1405-1411.

7. Malde S, Nambiar A, Umbach R, et al. Systematic review of the performance of noninvasive tests in diagnosing bladder outlet obstruction in men with lower urinary tract symptoms. Eur Urol 2017;71:391-402.

8. Levin RM, Haugaard N, O'Connor L, et al. Obstructive response of human bladder to BPH vs. rabbit bladder response to partial outlet obstruction: a direct comparison. Neurourol Urodyn 2000;19:609-629.

9. Bright E, Pearcy R, Abrams P. Ultrasound estimated bladder weight in men attending the uroflowmetry clinic. Neurourol Urodyn 2011;30:583-586.

10. Ghoniem GM, Regnier CH, Biancani P, Johnson L, Susset JG. Effect of vesical outlet obstruction on detrusor contractility and passive properties in rabbits. J Urol 1986;135:1284-1289.

11. Oelke M, Khullar V, Wijkstra H. Review on ultrasound measurement of bladder or detrusor wall thickness in women: techniques, diagnostic utility and use in clinical trials. World J Urol 2013;31:1093-1104.

12. Khullar V, Salvatore S, Cardozo L, Bourne TH, Abbott D, Kelleher C. A novel technique for measuring bladder wall thickness in women using transvaginal ultrasound. Ultrasound Obstet Gynecol 1994;4:220-223.

13. Oelke M, Wijkstra H. Ultrasound detrusor wall thickness measurements to diagnose bladder outlet obstruction in men. Urodinamica 2006;16:343-352.

14. Kojima M, Inui E, Ochiai A, Naya Y, Ukimura O, Watanabe H. Ultrasonic estimation of bladder weight as a measure of bladder hypertrophy in men with infravesical obstruction: a preliminary report. Urology 1996;47:942-947.

15. McLean GK, Edell SL. Determination of bladder volumes by gray scale ultrasonography. Radiology 1978;128:181182.

16. Carter S, Tubaro A. Standardisation of bladder ultrasonography. ICS subcomitee for standardization of imaging; 2001.

17. Watanabe Y. A method for volume estimation by using vector areas and centroids of serial cross-sections. IEEE Trans Biomed Eng 1982;29:202-205.

18. MATLAB and Statistics Toolbox Release 2012b, The MathWorks, Inc., Natick, Massachusetts, United States.

19. Oelke M, Mamoulakis C, Ubbink D, de la Rosette JJ, Wijkstra H. Manual versus automatic bladder wall thickness measurements: a method comparison study. World J Urol 2009;27:747-753. 